\title{
Aortic intima-media thickness can be used to determine target organ damage in adult patients with coronary artery disease risk factors
}

\author{
Atilla Bulut ${ }^{1}$, Armağan Acele ${ }^{1}$, Yurdaer Donmez ${ }^{1}$, Burcak Cakır Pekoz ${ }^{2}$, Murat Erdogan³, \\ Hilmi Erdem Sumbul', Yahya Kemal Icen ${ }^{1}$, Mevlut Koc ${ }^{1}$
}

\author{
${ }^{1}$ Department of Cardiology, University of Health Sciences-Adana Health Practice and \\ Research Center, Adana, Turkey \\ ${ }^{2}$ Department of Radiology, University of Health Sciences-Adana Health Practice and \\ Research Center, Adana, Turkey \\ ${ }^{3}$ Department of Internal Medicine, University of Health Sciences-Adana Health \\ Practice and Research Center, Adana, Turkey
}

Submitted: 22 June 2019

Accepted: 1 July 2019

Arch Med Sci Atheroscler Dis 2019; 4: e183-e190

DOI: https://doi.org/10.5114/amsad.2019.87002

Copyright $\odot 2019$ Termedia \& Banach

\section{Abstract}

Introduction: We aimed to evaluate the relationship between abdominal aortic intima-media thickness (AA-IMT) and thoracic aortic intima-media thickness (TA-IMT) values and to investigate their relationship with common carotid intima-media thickness (CC-IMT) in patients with coronary artery disease (CAD) risk factors.

Material and methods: This study included 100 patients who underwent transesophageal echocardiography (TEE) examination for different reasons with at least one CAD risk factor. CC-IMT, AA-IMT, and TA-IMT values were measured. Patients with CC-IMT >0.9 mm were considered as having increased CC-IMT. Patients were divided into two groups with and without increased CC-IMT.

Results: Mean AA-IMT and TA-IMT values of all patients were $1.55 \pm 0.27 \mathrm{~mm}$ and $1.39 \pm 0.25 \mathrm{~mm}$, respectively. In patients with increased CC-IMT, blood pressure, hyperlipidemia and hypertension frequency, creatinine, total and low-density lipoprotein ( $L D L)$ cholesterol, triglyceride, high-sensitivity C-reactive protein (hs-CRP) and uric acid levels, AA-IMT and TA-IMT values were higher, while the high-density lipoprotein $(\mathrm{HDL})$ cholesterol level was lower than the normal CC-IMT group. AA-IMT, systolic blood pressure (SBP), total and HDL cholesterol levels independently determined the patients with CCIMT $>0.9 \mathrm{~mm}$. Each $0.1 \mathrm{~mm}$ increase in AA-IMT value was found to increase the probability of having CC-IMT $>0.9 \mathrm{~mm}$. In addition, linear regression analysis showed that CC-IMT was closely and independently related to AAIMT $(p<0.001$ and $\beta=0.599)$. When the cut-off value for AA-IMT was taken as $1.5 \mathrm{~mm}$, it determined the patients with CC-IMT $>0.9 \mathrm{~mm}$ with $86 \%$ sensitivity and $82 \%$ specificity.

Conclusions: The AA-IMT value was found to be higher than TA-IMT in the same patient. Also it was found that AA-IMT was more closely related to CC-IMT.

Key words: intima-media thickness, thoracic and abdominal aorta, coronary artery disease risk factors.
Corresponding address: Prof . Dr. Mevlut Koc Department of Cardiology University of Health Sciences Adana Health Practice and Research Center Dr. Mithat Özsan Bulvarı Kışla Mah. 4522 Sok. No: 1 Yüreğir, Adana Turkey Phone/fax:

+ (90) 5062425989

E-mail: mevlutkoc78@yahoo.com 


\section{Introduction}

The atherosclerotic process starts at the tunica intima and tunica media layer in the arterial wall and causes a structural change in the early period as increased intima-media thickness (IMT). Increased IMT of the posterior arterial wall can be detected clearly with ultrasonography (US). In the evaluation of IMT in adulthood the main carotid IMT (CC-IMT) was evaluated with carotid US (C-US) and abdominal aortic IMT (AA-IMT) obtained by abdominal US (A-US) is used to evaluate the signs of atherosclerosis in childhood [1, 2]. IMT is used for early detection of macrovascular complications in both age groups. AA-IMT measurement can be used to detect the development of early atherosclerosis due to the initial onset of atherosclerosis in the distal abdominal aorta [3-5]. There are limited studies regarding the usage of $A A-I M T$ in patients with coronary artery disease (CAD) risk factors [6-11].

However, recently published articles have reported that AA-IMT may be easily measured with high-resolution devices [8-11]. Another important and atherosclerotic process-related IMT measurement region is thoracic aortic IMT (TA-IMT). However, with US devices, this region cannot be evaluated because of its bone structure. TA-IMT measurement with transesophageal echocardiography (TEE) has been performed successfully and objectively for many years, and increased IMT has been associated with advanced atheroma formation, poor prognosis, and early atherosclerosis detection [12-15].

There are many studies which are about AA-IMT and TA-IMT measurements in different patient groups, but there is no study with both AA-IMT and TA-IMT measurements in the same patient group. The aorta has ascending, descending thoracic, and abdominal parts anatomically. The in tima layer is seen throughout the aorta. It is still not known which section of the aorta has a higher IMT value and which part of the aortic IMT value is related to CC-IMT.

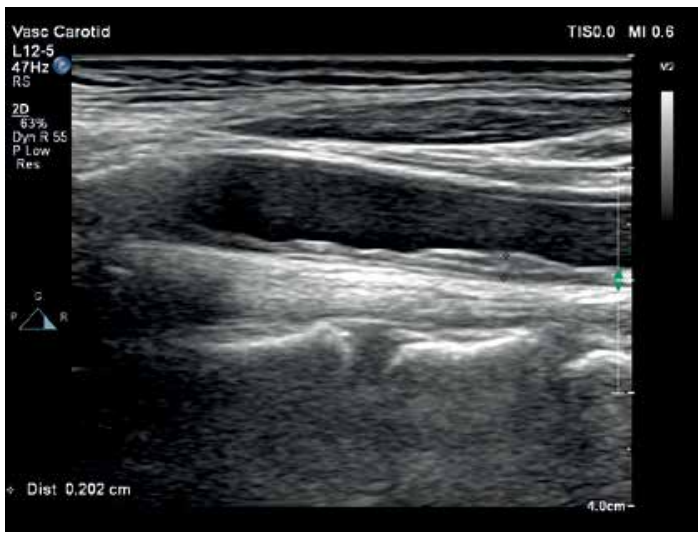

Figure 1. Patients with known CAD or atheroma (CC-IMT > $1.5 \mathrm{~mm}$ ) were excluded
Our aim was to assess AA-IMT and TA-IMT values obtained by A-US and TEE, respectively, and to investigate their relationship with CC-IMT in patients with CAD risk factors.

\section{Material and methods}

\section{Study population}

This cross sectional study included 100 patients who underwent TEE examination for different reasons with at least one CAD risk factor. The subjects were divided into two groups as 50 patients with increased CC-IMT (mean age: 52.8 \pm 7.3 years, male/female: $30 / 20$ ) and 50 patients with normal CC-IMT values (mean age: 50.2 \pm 10.1 years, male/female: $37 / 13$ ). Patients with known CAD or CC, TA and AA atheroma (CC-IMT $>1.5 \mathrm{~mm}$, TA-IMT > $3.00 \mathrm{~mm}$, AA-IMT > $3.00 \mathrm{~mm}$ ) were excluded (Figure 1). Nineteen patients with known carotid and aortic atheroma or plaque (C-IMT > $1.5 \mathrm{~mm}$ ) were excluded from the study. Patients with previous aortic operations, secondary or malignant hypertension, pulmonary hypertension, abdominal aortic aneurysm or dissection, congestive heart failure, cerebrovascular disease, moderate-severe valvular heart disease, inflammatory, hematologic diseases, cancer, thyroid disease, moderate-severe liver and kidney disease, pregnancy, active infectious disease, or age under 18 years old were excluded. The Local Ethics Committee approved the study protocol. All patients included in the study were informed about the study and provided their written informed consent.

Detailed physical examination was performed and thorough medical history was taken. Demographic characteristics, systolic and diastolic blood pressure (DBP), and heart rate were recorded. Patients were questioned about the presence of smoking, hypertension, diabetes mellitus, hypercholesterolemia, CAD, and obesity. Body mass index values were calculated. All medications related to CAD risk factors were noted.

\section{Laboratory measurements}

Venous blood samples were obtained at polyclinic admission. Samples were taken from the cubital vein into blood tubes. Blood counts were measured by a Sysmex K-1000 (Block Scientific, Bohemia, New York) auto analyzer within 5 min of sampling. Serum glucose, blood urea nitrogen, creatinine, total cholesterol, low-density lipoprotein $(\mathrm{LDL})$ cholesterol, high-density lipoprotein (HDL) cholesterol, triglyceride, high-sensitivity C-reactive protein (hs-CRP) and uric acid concentrations were measured with an automated chemistry analyzer (Abbott Aeroset, Minnesota, USA) using commercial kits (Abbott). 
Intima-media thickness measurement of the common carotid artery and abdominal aorta

The common carotid artery and abdominal aorta were examined using a high-resolution US device (Philips EPIQ 7) equipped with a linear (12 $\mathrm{MHz})$ and convex $(5 \mathrm{MHz})$ high-resolution transducer (Philips Health Care, Bothell, WA, USA). All arteries were evaluated both longitudinally and transversely. The ultrasound scanner setting was made to be useful for every patient for all B-mode USG examinations (gain 55-75 dB; penetration depth 2.5-16 cm; dynamics range 50-60 and zoom range 0.8-2.0). Analyses were performed on 2 high-quality images. IMT was defined as the distance between front edges of the first and second echogenic lines. The first line represents the intima and lumen border and the second line represents the collagen layer of the adventitia. Two independent and blind observers measured vascular IMTs. All IMT values were calculated as the average of 6 measurements. Patients were in a supine position, and they turned their head to the $45^{\circ}$ opposite direction of the examined carotid artery. CC-IMT was measured from the posterior wall of the carotid artery's pre-bifurcation segment (10-20 mm) (Figure 2). A-IMT was examined at the segment between the renal artery and iliac artery bifurcation. Also, the posterior wall of the abdominal aorta was used for the AA-IMT measurement (Figure 3). CC-IMT values higher than $>0.9 \mathrm{~mm}$ were accepted as increased [16].

\section{Intima-media thickness measurement of the descending thoracic aorta}

Transesophageal echocardiography examinations were performed with the EPIQ 7 (Philips Healthcare Andover MA, USA). This procedure was applied after $8 \mathrm{~h}$ of fasting and the patient was deeply sedated by an anesthesiologist. The TEE

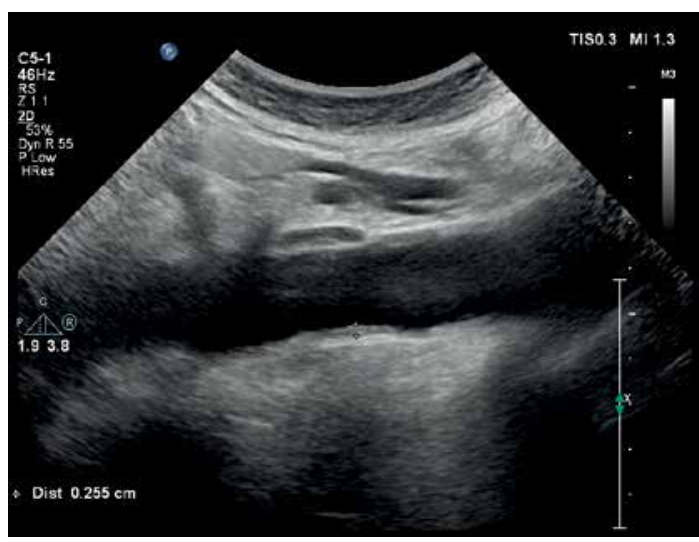

Figure 3. Increased abdominal aortic intima-media thickness $(2.6 \mathrm{~mm})$ in abdominal B-mode ultrasound probe was a multiplane transesophageal transducer. Patients were positioned in the left lateral decubitus position. The probe was engaged to the esophageal and gastric entry level. The experienced cardiology specialists were blind regarding patient details. All patients well tolerated the TEE procedure and there was no complication. TA-IMT was measured from 6 segments of the thoracic aorta. These segments were: 1) ascending aorta, 2) arch, 3) from 0 to $5 \mathrm{~cm}$ distal to the arch, 4) from 5 to $10 \mathrm{~cm}$ distal to the arch, 5) from 10 to $15 \mathrm{~cm}$ distal to the arch, and 6) from 15 to $20 \mathrm{~cm}$ distal to the arch. Mean of the 12 measurements (2 for any segment) was accepted as TA-IMT [15]. TA-IMT was defined as the distance from the leading edge of the lumen-intima interface to the leading edge of the media-adventitia interface of the posterior or far wall (Figure 4).

\section{Statistical analysis}

All analyses were done in SPSS 22.0 (IBM SPSS Statistics Version 22.0. Armonk, NY: IBM Corp.) software. Continuous variables and categorical variables were expressed as mean \pm standard deviation and numbers and percentages, respectively. The $\kappa$ coefficient was used to examine the

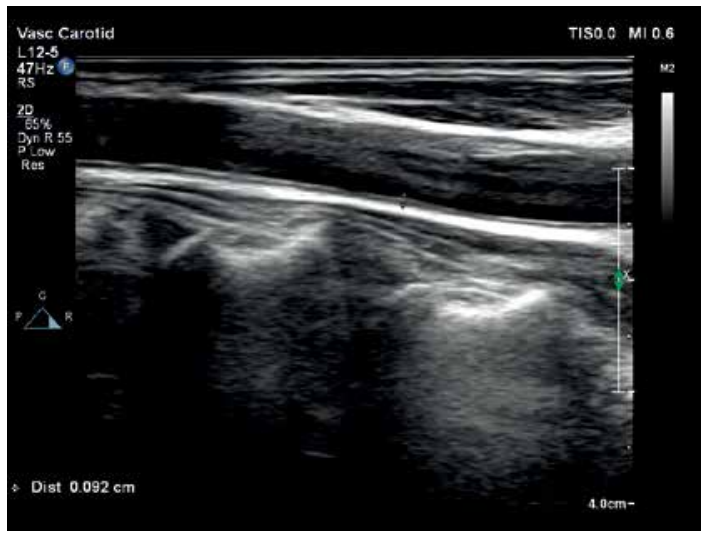

Figure 2. Increased carotid intima-media thickness $(0.92 \mathrm{~mm})$ in carotid B-mode ultrasound

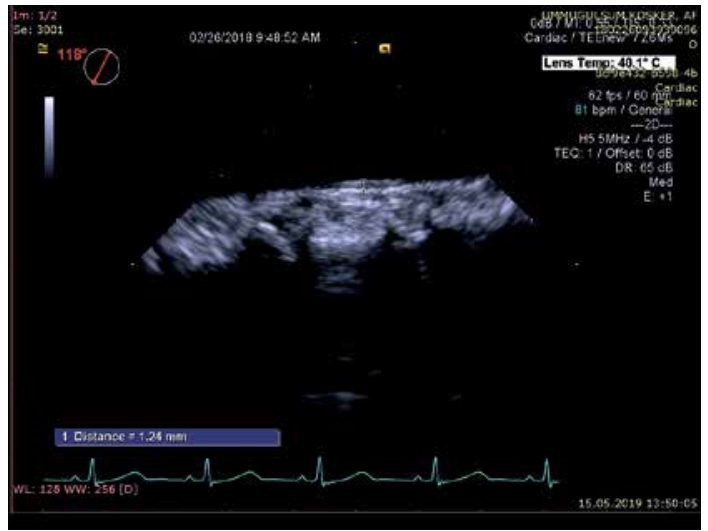

Figure 4. Thoracic aorta intima-media thickness in transesophageal echocardiography $(1.24 \mathrm{~mm})$ 
interobserver variability of all the IMT measurements. The Kolmogorov-Smirnov test was used to test the normality of continuous variables. Student's $t$ test was used for the normal distributed variables. The Mann-Whitney test was used for the variables without normal distribution. Categorical variables were analyzed by the chisquare $\left(\chi^{2}\right)$ test. Multivariate linear regression analysis was performed to evaluate the CC-IMT and AA-IMT relationship. Also, logistic regression analysis was used to detect the parameters which independently determine the patients with CC-IMT $>0.9 \mathrm{~mm}$. Receiver operating curve (ROC) analysis was done. $P<0.05$ was accepted as statistically significant.

\section{Results}

AA-IMT and TA-IMT measurements were successfully obtained from all patients included in the study after exclusion criteria. Mean, median, minimum and maximum AA-IMT values of all patients were $1.55 \pm 0.27,1.60,1.00$ and $3.00 \mathrm{~mm}$, respectively. Mean, median, minimum and maximum TA-IMT values of all patients were 1.39 $\pm 0.25,1.38,0.85$ and $2.50 \mathrm{~mm}$, respectively. Mean, median, minimum and maximum $\Delta$ aortic IMT values (difference between AA-IMT and TA-IMT) of all patients were $0.16 \pm 0.15,0.14,-0.17$ and $0.53 \mathrm{~mm}$, respectively. Cohen $\kappa$ values that evaluate interobserver and intraobserver variability were over $90 \%$ for all IMT measurements. AA-IMT

Table I. Demographic, clinic and laboratory findings in patients with increased and normal carotid intima-media thickness

\begin{tabular}{|c|c|c|c|}
\hline Parameter & Increased C-IMT & Normal C-IMT & $P$-value \\
\hline Age [years] & $52.8 \pm 7.3$ & $50.2 \pm 10.1$ & 0.139 \\
\hline Gender (male) & 30 & 37 & 0.202 \\
\hline Heart rate [beats/min] & $77.3 \pm 7.1$ & $75.9 \pm 5.8$ & 0.283 \\
\hline Office systolic BP [mm Hg] & $130 \pm 11$ & $122 \pm 9$ & $<0.001$ \\
\hline Office diastolic BP [mm Hg] & $83 \pm 10$ & $79 \pm 10$ & 0.017 \\
\hline Body mass index $\left[\mathrm{kg} / \mathrm{m}^{2}\right]$ & $28.1 \pm 2.9$ & $27.0 \pm 3.6$ & 0.120 \\
\hline Smoking, $n(\%)$ & $25(50 \%)$ & $19(38 \%)$ & 0.147 \\
\hline Diabetes mellitus, $n$ (\%) & $10(20 \%)$ & $14(28 \%)$ & 0.401 \\
\hline Hypercholesterolemia, $n$ (\%) & $23(46 \%)$ & $10(20 \%)$ & 0.033 \\
\hline Hypertension, $n$ (\%) & $28(56 \%)$ & 17 (34\%) & 0.009 \\
\hline Obesity, $n$ (\%) & $12(24 \%)$ & $9(18 \%)$ & 0.624 \\
\hline ACEI and/or ARB, $n(\%)$ & $40(80 \%)$ & 45 (90\%) & 0.205 \\
\hline Beta blocker, $n$ (\%) & 35 (70\%) & $30(60 \%)$ & 0.168 \\
\hline Diuretic therapy, $n$ (\%) & $21(39 \%)$ & $105(41 \%)$ & 0.879 \\
\hline Calcium channel blocker, $n$ (\%) & $2(4 \%)$ & 7 (14\%) & 0.160 \\
\hline Statin therapy, $n$ (\%) & $37(74 \%)$ & 35 (70\%) & 0.895 \\
\hline Glucose [mg/dl] & $132 \pm 46$ & $122 \pm 35$ & 0.089 \\
\hline BUN [mg/dl] & $29.7 \pm 8.5$ & $28.6 \pm 7.3$ & 0.491 \\
\hline Creatinine $[\mathrm{mg} / \mathrm{dl}]$ & $0.85 \pm 0.25$ & $0.77 \pm 0.16$ & 0.043 \\
\hline Total cholesterol [mg/dl] & $205 \pm 51$ & $181 \pm 37$ & 0.009 \\
\hline LDL cholesterol [mg/dl] & $136 \pm 37$ & $118 \pm 33$ & 0.013 \\
\hline HDL cholesterol [mg/dl] & $37.7 \pm 9.1$ & $42.4 \pm 9.2$ & 0.012 \\
\hline Triglyceride [mg/dl] & $200 \pm 78$ & $141 \pm 41$ & $<0.001$ \\
\hline hs-CRP [mg/l] & $1.28 \pm 1.33$ & $0.59 \pm 0.65$ & 0.002 \\
\hline Uric acid [mg/dl] & $5.07 \pm 0.99$ & $4.67 \pm 0.88$ & 0.037 \\
\hline
\end{tabular}

$B P$ - blood pressure, $A R B$ - angiotensin receptor blockers, $A C E I$ - angiotensin converting enzyme inhibitors, BUN - blood urea nitrogen, $H D L$ - high-density lipoprotein, hs-CRP - high sensitivity C-reactive protein, LDL - low-density lipoprotein, IMT-intima-media thickness. 
values were higher than TA-IMT in all patients except 11 patients. The study population was divided into two groups as increased CC-IMT (CC-IMT $>0.9 \mathrm{~mm})$ and normal CC-IMT (CC-IMT (0.9) according to CC-IMT values and all parameters were compared. Demographic, clinical, laboratory and IMT values of study groups were compared. When the demographic and clinical data of both groups were evaluated, the prevalence of hypercholesterolemia and HT, SBP and DBP was found to be higher in patients with increased CC-IMT, and other demographic and clinical parameters were similar between the two groups (Table I).

Creatinine, total cholesterol, LDL cholesterol, triglyceride, hs-CRP, uric acid, CC-IMT, AA-IMT, and TA-IMT values were significantly higher and HDL cholesterol levels were significantly lower in patients with increased CC-IMT (Table I).

AA-IMT and TA-IMT values were significantly higher in patients with increased CC-IMT (Table II). In the logistic regression analyses AA-IMT, systolic blood pressure, total and HDL cholesterol levels independently determined the patients with increased CC-IMT (CC-IMT >0.9 mm). According to this analysis, it was found that every $0.1 \mathrm{~mm}$ increase in AA-IMT value doubled the probability of having increased CC-IMT (Table III). In addition, linear regression analysis showed that CC-IMT values were closely and independently related to AAIMT ( $p<0.001$ and $\beta=0.599$, Figure 5).

ROC curve analysis was also performed to identify patients with CC-IMT $>0.9 \mathrm{~mm}$; the parameters AUC $>0.700$ are shown in Table IV. The area under the ROC curve was maximum for AAIMT and the area under the curve was found to be $0.843,0.785$ and 0.757 for AA-IMT, TA-IMT and SBP respectively. A cut-off value of $1.5 \mathrm{~mm}$ for $\mathrm{AA}$ IMT detected increased CC-IMT with $86 \%$ sensitivity and $82 \%$ specificity (Figure 6).

\section{Discussion}

The most important finding of our study was that the AA-IMT value was significantly higher than TA-IMT in patients with CAD risk factors. To the best of our knowledge, this was the first study in which AA-IMT and TA-IMT were determined in the same patient group at the same time. Another important finding of our study was that the AA-IMT value was significantly and more closely related to increased CC-IMT than the TA-IMT value. When the AA-IMT cut-off value was taken as $1.5 \mathrm{~mm}$, it determined the patients with increased CC-IMT with acceptable sensitivity and specificity.

Table II. Vascular ultrasound findings in patients with increased and normal carotid intima-media thickness

\begin{tabular}{|lccc|}
\hline IMT & Increased C-IMT & Normal C-IMT & $P$-value \\
\hline Common carotid IMT [mm] & $1.20 \pm 0.17$ & $0.74 \pm 0.12$ & $<0.001$ \\
\hline Thoracic aorta IMT [mm] & $1.54 \pm 0.19$ & $1.23 \pm 0.22$ & $<0.001$ \\
\hline$\Delta$ Aort IMT [mm] & $0.16 \pm 0.17$ & $0.17 \pm 0.12$ & 0.804 \\
\hline Abdominal aort IMT [mm] & $1.70 \pm 0.18$ & $1.40 \pm 0.25$ & $<0.001$ \\
\hline
\end{tabular}

IMT - intima-media thickness.

Table III. According to multivariate regression analysis, independent risk factors for occurrence of increased carotid intima-media thickness

\begin{tabular}{|lccc|}
\hline Variable & Odds ratio & 95\% confidence interval & $P$-value \\
\hline Abdominal aort IMT $(0.1 \mathrm{~mm})$ & 1.986 & $1.515-2.604$ & 0.001 \\
\hline Office systolic BP $(1 \mathrm{~mm} \mathrm{Hg})$ & 1.315 & $1.146-1.510$ & 0.002 \\
\hline Total cholesterol $[\mathrm{mg} / \mathrm{dl}]$ & 1.020 & $1.008-1.032$ & 0.012 \\
\hline HDL cholesterol $[\mathrm{mg} / \mathrm{dl}]$ & 0.910 & $0.862-0.961$ & 0.026 \\
\hline
\end{tabular}

$B P$ - blood pressure, IMT - intima-media thickness, HDL - high-density lipoprotein.

Table IV. ROC curve analysis of abdominal aort intima-media thickness and office systolic blood pressure for predicting increased carotid intima-media thickness

\begin{tabular}{|lccccc|}
\hline Variable & Area under ROC curve & P-value & Cut-off value & Sensitivity (\%) & Specificity (\%) \\
\hline Abdominal aort IMT & $0.843(0.765-0.922)$ & $<0.001$ & $1.50 \mathrm{~mm}$ & 86 & 72 \\
\hline Thoracic aorta IMT & $0.785(0.750-0.920)$ & $<0.001$ & $1.40 \mathrm{~mm}$ & 76 \\
\hline Office systolic BP & $0.757(0.657-0.857)$ & $<0.001$ & $124 \mathrm{~mm} \mathrm{Hg}$ & 80 & 78 \\
\hline
\end{tabular}

IMT - intima-media thickness, BP-blood pressure 


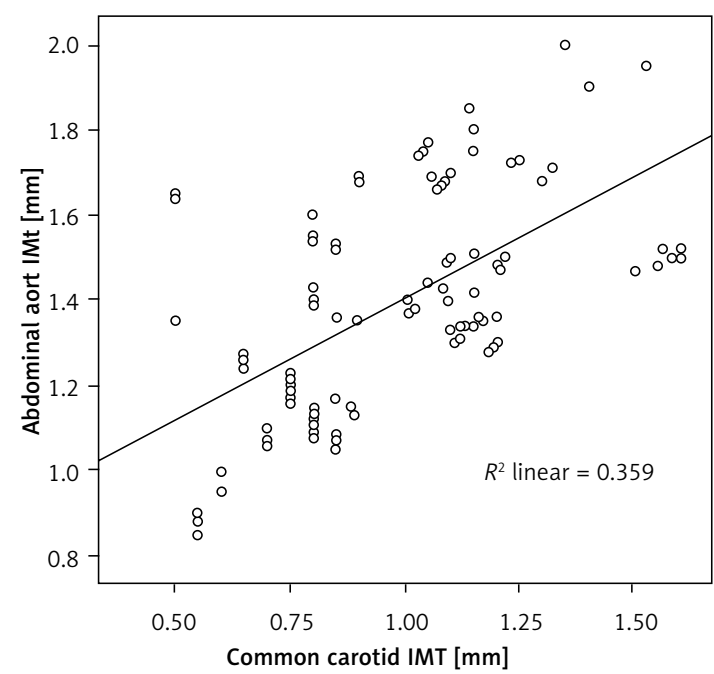

Figure 5. Simple scatter/dot diagram for the relationship between the main carotid and thoracic aortic intima-media thickness: a close relationship between the main carotid and thoracic aortic intima-media thickness

CAD risk factors: i) increase smooth muscle cell proliferation in the vessels, ii) cause oxidative stress in tissues, iii) thicken the intima-media layer of the vessel with increasing free oxygen radicals [2, 16-19]. Regular CC-IMT evaluation is advised to detect the subclinical atherosclerosis in patients with CV risk factors such as hypertension, diabetes, hyperlipidemia, smoking, advanced age, and obesity [16-19]. There are many studies which have shown a close relation between CC-IMT and CAD, myocardial infarction, and stroke. Therefore, CC-IMT evaluation is advised as a routine screening method [13, 16-19]. Carotid artery evaluation is a preferred method, because it is superficially located, easy to find and easy to visualize. TA-IMT can be easily measured during the TEE evaluation, and it can be used for detection of early atherosclerosis and as a follow-up method for CV diseases [12-15]. However, TEE is invasive and it can be used in a limited number of patients; it cannot be used in routine practice of patients with CV risk factors. Moreover, it only can be useful for a limited patient group who need an evaluation with TEE. The abdominal aorta is another important IMT measurement location. It is a location in which the first atherosclerotic lesions and early signs of atherosclerosis such as fatty streaks can be seen. A limited number of studies have mentioned the importance of AA-IMT as a screening method for detection of early atherosclerosis in adults [6-11, 14, 15]. Moreover, the abdominal aorta is the first area to be affected by atherosclerosis in pediatric patients with $\mathrm{CV}$ risk factors. So, AA-IMT is the first choice in childhood. The main limiting factors for AA-IMT evaluation are abdominal fat tissue and usage of low pen-

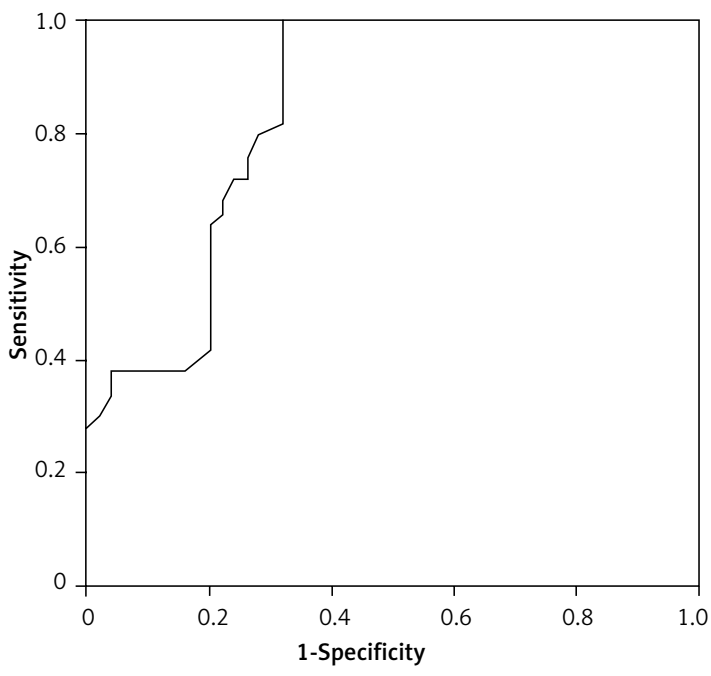

Figure 6. ROC curve for thoracic aortic intima-media thickness for determination of increased carotid intima-media thickness

etrating US devices [6]. However, it is shown that new high-resolution US devices and probes made possible reliable AA-IMT measurements [8-11].

Due to these limitations and difficulties in the measurement of AA-IMT and TA-IMT, we did not find any study comparing the TA-IMT and AA-IMT values in the same patient group and measuring IMT from both regions in the literature. In our study IMT was measured from both aortic anatomical regions for the first time. We found that AA-IMT values were higher than TA-IMT values. This finding supports the hypothesis that an increase in vascular IMT was initially initiated from AA. Another important finding was the comparison of CC-IMT ant the IMT values obtained from the TA and AA. AA-IMT values were more closely related to CC-IMT. It was shown that AA-IMT could independently predict the patients with increased CC-IMT. Every $0.1 \mathrm{~mm}$ increase in AA-IMT value doubled the probability of having increased CCIMT (Table III). A cut-off value of $1.5 \mathrm{~mm}$ for AAIMT was shown to be potentially useful to detect patients with increased CC-IMT. Recent studies have also reported that a $1.5 \mathrm{~mm}$ cut-off value could be used to document increased AA-IMT in patients with hypertension and diabetes mellitus $[9,10]$. Abdominal US is a visualization method which can be used in many CV diseases. AA-IMT may have a future like CC-IMT as a screening method to detect early atherosclerosis or subclinical organ damage in patients with CV risk factors.

There are some important limitations of this study. We investigated the relation between AAIMT and TA-IMT in a cross-sectional study design. Our patient size was relatively small; nevertheless, we showed that there was a significant relation between increased AA-IMT and increased CC-IMT. The study could be more meaningful if it was done 
prospectively and with more patients. We excluded patients with known CAD and presence of atheroma in any vascular regions. Some of the patients included in our study have been taking medications which could have effects on IMT. This may have affected our results. A recent study reported that age could be very closely related to CC-IMT and therefore a limit value for CC-IMT should be specified according to age [20]. There is no study about A-IMT measurement but the C-IMT measurement can be measured automatically and semi-automatically with new software programs, resulting in a lower average value than the manual measurement $[21,22]$. This automatic measurement especially removes operator dependence and is more useful for repetitive measurements. However, our high-resolution device did not have this software program so we could not make this evaluation. In our study, if IMT could be measured automatically and semi-automatically, more objective and meaningful results could be obtained. We used only 0.9 $\mathrm{mm}$ as a cut-off value in all patients. In addition, our study was not a follow-up study and did not evaluate the prognosis of CV diseases. We used high-resolution US devices and probes. Therefore, it may not be possible to achieve similar results with low frequency and low-resolution devices.

In conclusion, AA-IMT values are higher than TA-IMT in patients with different CAD risk factors. Also AA-IMT is more closely related to CC-IMT than TA-IMT. According to our results, patients with an AA-IMT value $>1.5 \mathrm{~mm}$ should be closely followed. CC-IMT measurement is advised in hypertension, diabetes, and hyperlipidemia treatment guidelines. AA-IMT should also be a part of A-US, which is a screening method for these diseases, and AAIMT values should be reported in the A-US results.

\section{Conflict of interest}

The authors declare no conflict of interest.

\section{References}

1. McCloskey K, Vuillermin P, Ponsonby AL, Cheung M, Skilton MR, Burgner D. Aortic intima-media thickness measured by trans-abdominal ultrasound as an early life marker of subclinical atherosclerosis. Acta Paediatr 2014; 103: 124-30.

2. Lilje C, Cronan JC, Schwartzenburg EJ, et al. Intima-media thickness at different arterial segments in pediatric type 1 diabetes patients and its relationship with advanced glycation end products. Pediatr Diabetes 2018; 19: 450-6.

3. Shin PS, Kim DS. Histochemical studies of fetal arteries of Koreans with special reference to atherogenesis in adults. Yonsei Med J 1963; 4: 37-42.

4. Nakashima Y, Chen YX, Kinukawa N, Sueishi K. Distributions of diffuse intimal thickening in human arteries: preferential expression in atherosclerosis-prone arteries from an early age. Virchows Arch 2002; 441: 279-88.
5. Jarvisalo MJ, Jartti L, Nanto-Salonen K, et al. Increased aortic intima-media thickness: a marker of preclinical atherosclerosis in high-risk children. Circulation 2001; 104: 2943-7.

6. Astrand H, Rydén-Ahlgren A, Sundkvist G, Sandgren T, Länne T. Reduced aortic wall stress in diabetes mellitus. Eur J Vasc Endovasc Surg 2007; 33: 592-8.

7. Su Y, Liu W, Wang D, Tian J. Evaluation of abdominal aortic elasticity by strain rate imaging in patients with type 2 diabetes mellitus. J Clin Ultrasound 2014; 42: 475-80.

8. Icen YK, Koc AS, Sumbul HE. Coronary artery disease severity is associated with abdominal aortic intima-media thickness in patients with non-st-segment elevation myocardial infarction. Angiology 2019; 70: 561-6.

9. Sumbul HE, Koc AS. The abdominal aortic intima-media thickness increases in patients with primary hyperparathyroidism. Exp Clin Endocrinol Diabetes 2019; 127: 387-95.

10. Koc AS, Sumbul HE. Increased aortic intima-media thickness may be used to detect macrovascular complications in adult type II diabetes mellitus patients. Cardiovasc Ultrasound 2018; 16: 8.

11. Koc AS, Gorgulu FF, Donmez Y, Icen YK. There is a significant relationship between morning blood pressure surge and increased abdominal aortic intima-media thickness in hypertensive patients. J Med Ultrason 2018; 45: 597-603.

12. Harrington J, Pena AS, Gent R, Hirte C, Couper J. Aortic intima media thickness is an early marker of atherosclerosis in children with type 1 diabetes mellitus. J Pediatr 2010; 156: 237-41.

13. Heiss G, Sharrett AR, Barnes R, Chambless LE, Szklo M, Alzola C. Carotid atherosclerosis measured by B-mode ultrasound in populations: associations with cardiovascular risk factors in the ARIC study. Am J Epidemiol 1991; 134: 250-6.

14. Berenson G, Srinivasan S, Bao W, Newman WP $3^{\text {rd }}$, Tracy RE, Wattigney WA. Association between multiple cardiovascular risk factors and atherosclerosis in children and young adults. N Eng J Med 1998; 338: 1650-5.

15. Nishino M, Masugata H, Yamada Y, Abe H, Hori M, Kamada T. Evaluation of thoracic aortic atherosclerosis by transesophageal echocardiography. Am Heart J 1994; 127: 336-44.

16. Williams B, Mancia G, Spiering W, et al. 2018 ESC/ESH Guidelines for the management of arterial hypertension. Eur Heart J 2018; 39: 3021-104.

17. Rydén L, Grant PJ, Anker SD, et al. ESC Guidelines on diabetes, pre-diabetes, and cardiovascular diseases developed in collaboration with the EASD: the Task Force on diabetes, pre-diabetes, and cardiovascular diseases of the European Society of Cardiology (ESC) and developed in collaboration with the European Association for the Study of Diabetes (EASD). Eur Heart J 2013; 34: 3035-87.

18. Piepoli MF, Hoes AW, Agewall S, et al. 2016 European Guidelines on cardiovascular disease prevention in clinical practice: The Sixth Joint Task Force of the European Society of Cardiology and Other Societies on Cardiovascular Disease Prevention in Clinical Practice (constituted by representatives of 10 societies and by invited experts) Developed with the special contribution of the European Association for Cardiovascular Prevention \& Rehabilitation (EACPR). Eur Heart J 2016; 37: 2315-81.

19. Catapano AL, Graham I, De Backer G, et al.; ESC Scientific Document Group. 2016 ESC/EAS Guidelines for the Management of Dyslipidaemias. Eur Heart J 2016; 37: 2999-3058. 
20. Koc AS, Sumbul HE. In determining cut-off values for increased carotid intima-media thickness age should be considered. Turk Kardiyol Dern Ars 2019; 47: 301-11.

21. Novo G, Di Miceli R, Orlando D, et al. Carotid intima-media thickness measurement through semi-automated detection software and analysis of vascular walls. Int Angiol 2013; 32: 349-53.

22. Shenouda N, Proudfoot NA, Currie KD, Timmons BW, MacDonald MJ. Automated ultrasound edge-tracking software comparable to established semi-automated reference software for carotid intima-media thickness analysis. Clin Physiol Funct Imaging 2018; 38: 396-401. 